\section{Neuroanatomical profile of hemineglect in patient's body image modification}

\author{
Maurizio Falso* and Eleonora Cattaneo Psy \\ Section of Neurological Rehabilitation, Clinical Institute Città di Brescia, Brescia, Italy
}

\section{Abstract}

Background: NSU is generally caused by right cerebral hemisphere lesions with a preeminent localization on the frontoparietal lobe.

Aim: To assess the correlation between the typology and the brain lesion site and the consensual consent modality of body image modification after an integrated rehabilitative and neuropsychological treatment.

Setting: A rehabilitation institute for the treatment of neurological gait disorders and neuropsichological failures.

Methods: Patients recruited were divided according to the brain lesion site into 3 groups $(I G=$ ischemic group $=5$ patients; $H G=$ hemorrhagic group $=4$ patients; $I G+H G=$ ischemic + hemorrhagic group $=3$ patients) based on CT brain performed in the post-acute phase. At time T0, the patients recruited underwent a systematic review of their current neuroradiological profile (location delineation and type of brain injury) compared with a consensual framing of the neuromotor and neuropsychological profile acquired at the time of taking charge in the ward. At time $\mathrm{T} 1$ and after the drafting and implementation of the rehabilitation treatment plan foreseen in the study ( 1 to 4 months after T0), the patients in our sample underwent a re-evaluation of their neuromotor and neuropsychological profile with controls of the same outcome parameters considered at time TO

Results: A parametrically but not statistically significant modification of the results obtained was observed by measuring the MI ULI, MI LLI and TCT scales in the group with hemorrhagic brain injury; the analysis of variance did not show any statistical significance in the relationship between the type of stroke (ischemic, hemorrhagic or both) and the motor impairment passing from time T0 to time T1. The analysis of variance did not reveal a statistically or parametrically significant relationship between the type (ischemic, hemorrhagic and ischemic + hemorrhagic) of cerebral stroke and the variations of the neuropsychological profile. The T-Student test showed statistically significant changes in the importance of the lesion site in defining the degree of motor disability. In particular, we observed, about the presence of frontal lobe lesions, a statistically significant variation passing from the T0 time to the T1 time for the following motor scales in 9 of 12 recruited patients: MI LLI $(26.4$ vs. 62, with $p<0.05)$, TCT (43.6 vs. 80.6, with $p<$ 0.01 for equal variance assumed and $p<0.05$ for equal variance not assumed), FAC (0.8 vs. 2.3 with $p<0,01$ for equal variance assumed and $p<0.05$ for equal variance not assumed).

Conclusion: We have confirmed the importance of the anatomical-dysfunctional correlation as a key concept from which to start in any neurorehabilitative treatment approach. Our work has highlighted the basic role of the right frontal lobe in the programming and execution of the gesture and its kinesthetic control as regards the left lower limb and the trunk.

\section{More Information \\ *Address for Correspondence: Dr. Maurizio Falso, MD, Physical Medicine and Rehabilitation Specialist, Section of Neurological Rehabilitation - Clinical Institute Città di Brescia - via Gualla 15 25128, Brescia, Italy, Tel: 349.4971729; Email: falsomaurizio@libero.it}

Submitted: 11 March 2020

Approved: 25 March 2020

Published: 26 March 2020

How to cite this article: Falso M, Psy EC. Neuroanatomical profile of hemineglect in patient's body image modification. J Nov Physiother Rehabil. 2020; 4: 001-008.

DOI: 10.29328/journal.jnpr.1001029

Copyright: (c) 2020 Falso M, et al. This is an open access article distributed under the Creative Commons Attribution License, which permits unrestricted use, distribution, and reproduction in any medium, provided the original work is properly cited.

Keywords: NSU; Body schema; Rehabilitative treatment of NSU

Abbreviations: NSU: Unilateral Spatial Neglect; MMSS: Mini Mental Status Scale; MI: Motiricity Index; TCT: Trunk Control Test; FAC: Functional Ambulation Category; IG: Ischemic Group; HG: Hemorrhagic Group; ULI: Left Upper Limb; LLI: Left Lower Limb; VLSM: Voxel-based LesionSymptom Cerebral Mapping; RDHF: Representational Drawing of the Human Figure; NEG P: Personal Neglect

\section{Check for updates}

OPEN ACCESS

\section{Introduction}

In line with the so-called "embodiment concept", the human bodily experience is characterized by the immediate feeling that our body is localized in a certain position in space and that the self is localized within these body limits [1]. Recent research found out that terms like "body image" and "body schema" have been often confused or sometimes used as synonyms [2]. As long ago as 1935, Shildler [3] tried to underline the great conceptual difference between "body image and body schema": the first one indicates the conscious knowledge about our body (mental image) while the second one defines the tridimensional image that each of us has of his own body, made up of senses, schema and mental representations. In a recent review, Maravita et al. [4], refer to a wide discussed [5-7] but unclear definition of body schema, 
or body representation, to indicate such neural system whereby space coding for action is centered on updated and multisensory information about the body. All basic daily living activities of humans need perfect integrity and integration of these complex evolutionary and cognitive aspects of body representation. From a neuropsychological point of view, a distortion of a patient's body schema and body image can produce the well known NSU or Unilateral Spatial Neglect with a collateral alteration of the patient's motor and perceptive functional profile. NSU is generally caused by right cerebral hemisphere lesions with a preeminent localization on the frontoparietal lobe. Patients with neglect often err toward the right when asked to point their hand straight ahead of the body midline. This particular orientation bias can be associated with a rotation of internal representation of the midsagittal plane of the body [8]. An interesting study of Biyu, et al. of the Washington University School of Medicine [9], demonstrated the relevant role of two neural networks that hold an updated map of body shape and posture localized in the ventral and dorsal frontoparietal cerebral areas. In this way, they underlined the central role of the white cerebral matter tracts connecting them (if disconnected, the functional impairment secondary to neglect was more severe than the isolated lesion of every single track). Even if some studies support the critical role of the ventral cerebral network (including the parietal lobule, the superior temporal gyrus, the temporoparietal junction and the white matter beneath the central sulcus), Ptak, et al. [10] underlined the important role of the dorsal cerebral attentional network as a potential functional outcome measure in NSU. In a particular way, the authors tried to define an anatomical-dysfunctional correlation in 29 patients affected by right hemisphere stroke with a secondary left hemineglect, by using the so-called "voxel-based lesion-symptom" cerebral mapping (VLSM). The neuroimage procedure used revealed a great correlation between the grade of motor impairment and the dorsal cerebral attentional network in patients affected by NSU. Moreover, a relevant role can be assumed by the temporoparietal cerebral junction, the dorsal premotor cortex, and the intraparietal sulcus as potential cerebral areas implicated in the appearance of spatial hemineglect. Many physicians tried to identify different cerebral areas involved in the neglect syndrome: temporal and inferior parietal cortex [11], the putamen and the internal capsule [12], the pont [13], the frontoparietal areas [14], the dorsal premotor cortex, the primary motor cortex, the somatosensorial area [15], the insula [16]. The rehabilitative approach in NSU is focused today on different techniques based on two well-known mechanisms: the "top-down" mechanism which includes spatial-motor and trunk rotational cues; the "bottom-up" mechanism which includes the eye-patching technique, prisms, vestibular caloric stimulation, inversed mirror, virtual reality, and neck vibration. These different rehabilitative approaches can be used not only alone but also in an integrated way to increase the final functional effect of the treatment course [17]. The purpose of the following study is to assess the correlation between the typology and the brain lesion site and the consensual consent modality of body image modification after an integrated rehabilitative and neuropsychological treatment in a sample of patients affected by NSU.

\section{Materials and methods}

\section{Participants}

In line with the aim of this study, we made e retrospective data analysis about a cohort of 12 patients affected by NSU (Unilateral Spatial Neglect) secondary to cerebral stroke (7 males, 5 females; mean age $60 \pm 2$ yy with an average amount of attended school years equal to $8.25 y \mathrm{y} \pm 2.83$ ) and hospitalized in the Rehabilitation Department of the Clinical Institute Città di Brescia; 5 of them were affected by cerebral ischemic stroke, 4 patients by a hemorrhagic stroke and 3 patients by an ischemic-hemorrhagic stroke. For the retrospective collection of data, we have received informed consent from the relatives of each individual patient hospitalized and included in our study. This procedure was conducted in accordance with the principles of the Helsinki Declaration. Once the informed consent has been collected, the Health Department has approved the retrospective data collection protocol necessary to demonstrate the purpose of our study.

\section{Inclusion criteria:}

- presence of a hemineglect profile

- CT evidence at time T0 of a right hemisphere stroke

- less than 1 month of inter-time between the cerebral stroke and the hospitalization

- $\quad$ MMSS score higher than 9

- Italian mother tongue

\section{Study design and outcome measures}

Patients recruited in the following study were divided according to the brain lesion type into 3 groups (IG = ischemic group $=5$ patients; $\mathrm{HG}=$ hemorrhagic group $=4$ patients; IG + $\mathrm{HG}=$ ischemic + hemorrhagic group $=3$ patients) based on CT brain evaluation performed in the post-acute phase.

At time T0 (day of access to the Neurological Rehabilitation Department of the Clinical Institute - Città di Brescia), the patients recruited underwent a systematic review of their current neuroradiological profile (location delineation and type of brain injury) compared with a consensual framing of the neuromotor (MI, TCT, FAC) and neuropsychological profile (RDHF, NEG P) acquired at the time of taking charge in the ward. At time T1 and after the drafting and implementation of the rehabilitation treatment plan foreseen in the study (1 to 4 months after T0), the patients in our sample underwent a reevaluation of their neuromotor and neuropsychological profile with controls of the same outcome parameters considered at time T0 (Figure 1). 


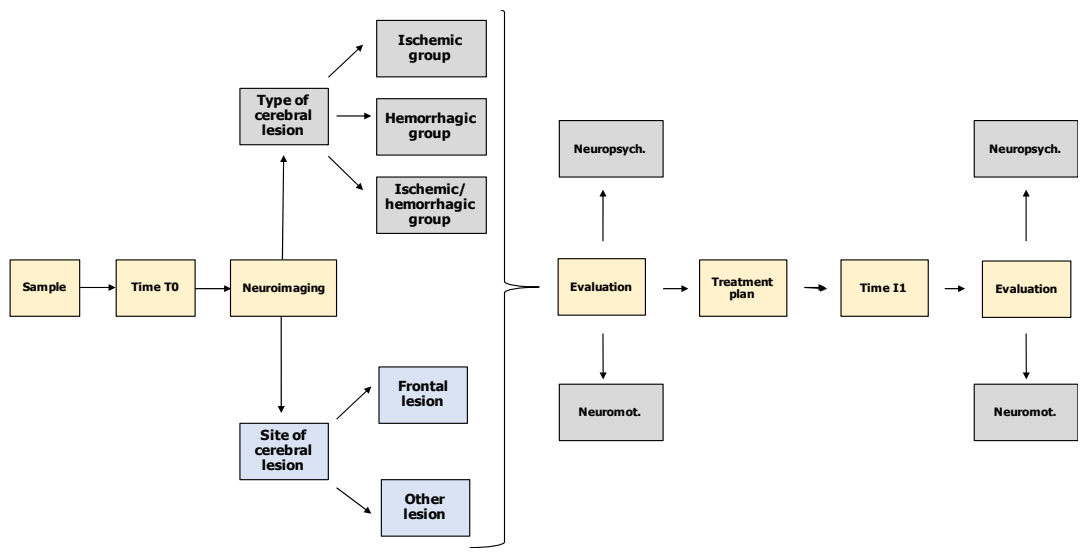

Figure 1: Resume of the study design (own elaboration).

\section{Outcome measures}

The outcome measures, used for the evaluation of the patients in our sample, investigated 2 aspects of NSU: neuromotor damage and neuropsychological damage.

- $\quad$ Evaluation setting of the neuromotor impairment

- Motricity Index (MI)(developed in 1990 by Collin C and Wade D)(an ordinal-type scale that quantify poststroke motor impairment; limb function is invastigated, on both sides, through the request for fundamental movements for the daily life activities; for the upper limb, the ability to perform a pinch grip, flexion of the elbow and abduction of the shoulder are analyzed; the ankle dorsiflexion, knee extension and hip flexion are examined for the lower limb);

- Trunk Control Test (TCT) )(developed in 1990 by Collin C and Wade D)(an ordinal scale that allows the assessment of balance and control of the trunk: the postural supine-seated passage, turn in the supine position, on the healthy side and on the affected side and maintain the sitting position without supports for 30 seconds; a score is assigned, differentiating between a movement that needs help from an assistant and a self-sufficient movement with or without the need to cling to the bed; the total sum of the scores can reach a maximum score of 100);

- Functional Ambulation Category (FAC)(developed in 1986 by Holden M.K. and colleagues)(a tool for measuring the ambulatory capacity; it's an ordinal scale comprising five classes.; this subdivision takes into account specific criteria, related to both the functionality of the path and the characteristics of the surface of the ground where it is practiced).

- Evaluation setting of the neuropsychological impairment

Representational drawing (RDHF)(developed in 1987 by Wilson) (subtest that consists of three tests: the patient is asked to draw a clock, then a human figure, and finally a butterfly on three different sheets, A4 size; the drawings are made to run on three different sheets; the score to be assigned is 1 for each drawing if it appears substantially symmetrical).

Personal Neglect evaluation scale (NEG P) (developed in 1992 by Zoccolotti and colleagues) (the examiner evaluates three tasks:1. The subject is given a comb (or brush) and is asked to comb: "Can you show me how to comb your hair?" 2. Subject-Woman: a powder case is supplied to the subject and is delivered to the subject asked to get inspired: "Does he shown me how to put the powder?" Subject-Man: a razor with a safety cap is provided and he is asked to shave: "Does he shown me how to use the razor?"; 3 . The subject is asked to wear glasses: "Does he shown me how to put on his glasses?"; the number of "blows" to the right and left must be counted in order to arrive at a judgment on the symmetry of execution of the task; the score reflects the level of asymmetry in the exploration of personal space: 0 is the absence of asymmetry, 1 mild, 2 moderate, 3 severe right-left asymmetry in the exploration of personal space; no feedback should be given on the correctness of the task or to encourage completion of the service).

\section{Neuroanatomical evaluation model of stroke patients}

For the aim of this study, CT brain evaluation was performed in each patient of our sample in the post-acute phase of their brain damage (at time T0, day of access to the Neurological Rehabilitation Department); starting from this evaluation, a systematic retrospective review of their current neuroradiological profile (location delineation and type of brain injury) (Table 1) was made, focusing attention on:

- type of cerebral lesion (ischemic, hemorrhagic or both) with the differentiation of 3 study groups (IG = ischemic group; $\mathrm{HG}=$ hemorrhagic group; IHG = ischemichemorrhagic group);

- anatomical localization of cerebral damage. 


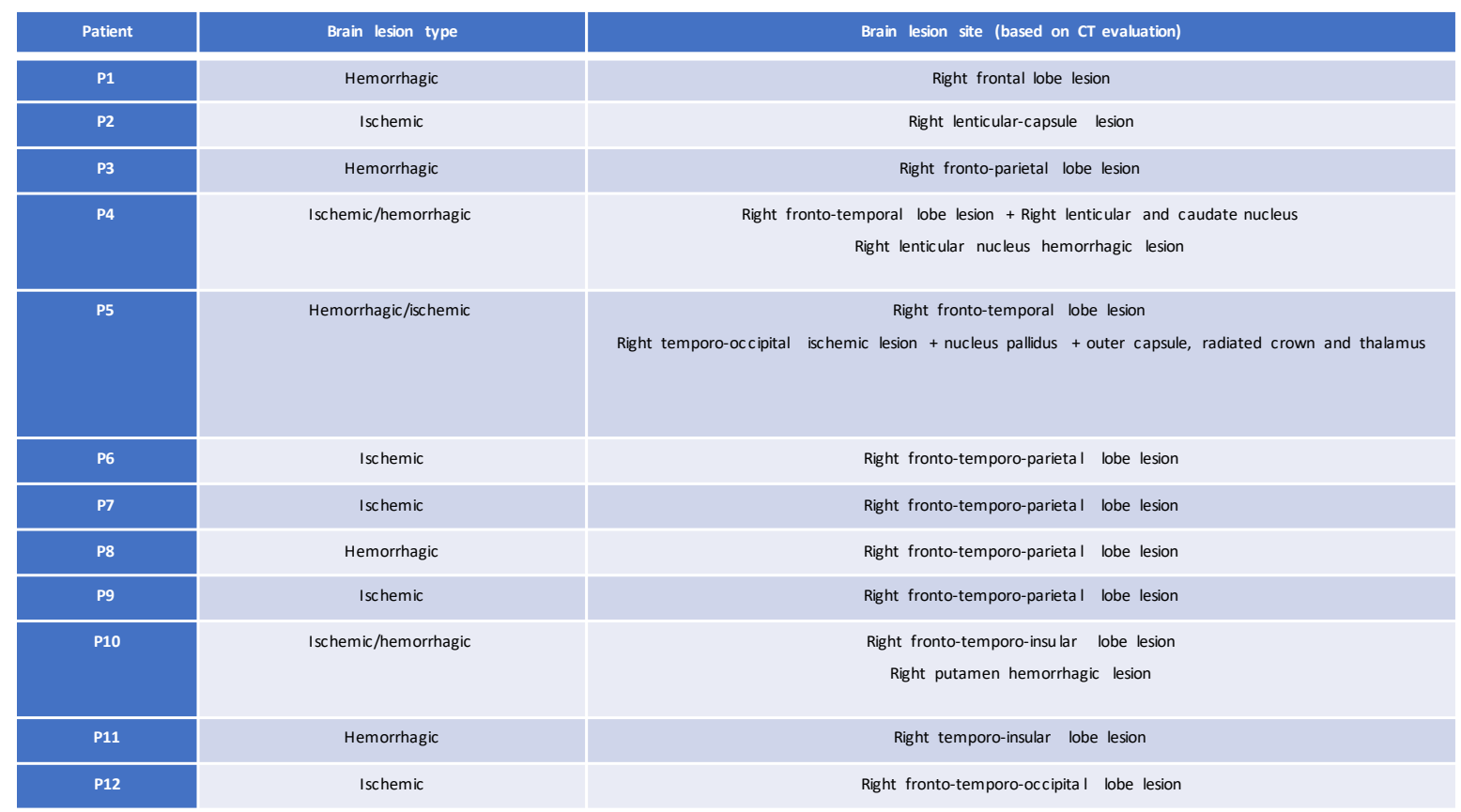

Table 1: Resume of the neuroanatomical profile based on CT evaluation of our study sample (own elaboration).

\section{Neurorehabilitative treatment procedure}

Recruited patients underwent an integrated rehabilitation treatment consisting of:

a) an individual rehabilitative project for each recruited patient with related functional goals

b) an individual rehabilitative exercises program for each recruited patient

c) a multi-professional rehabilitative project with the identification of all professional figures needed to realize our functional rehabilitative goals

\section{Neuropsychological treatment procedure}

Recruited patients underwent a neuropsychological rehabilitative treatment consisting of:

- a visual performance exploration

- perceptive and spatial mind exploration

- lecture training with visual cues positioned on the left side of the lecture page

- drawing copy on matrix points

- description of figures and real environments.

\section{Statistical analysis}

Data analysis was carried out using SPSS statistical software (version 22). The relationship between the typology of cerebrovascular accident and the degree of functional disability and alteration of the neuropsychological profile of our 12 patients was investigated through the use of analysis of variance at time $\mathrm{T} 0$ and $\mathrm{T} 1$ (mean $\mathrm{T} 1$ corresponding to approximately 2.5 months). The analysis of the data subsequently involved the use of the T-Student parametric test (considering statistically significant the variations of the test results over time with $p<0.05$ ) to investigate the relationship between the cerebral damage localization and neuromotor and neuropsychological time-related outcomes of 12 patients recruited. To perform this analysis, the sample was divided into 2 groups with the involvement of the frontal lobe, detected through the neuroradiological investigation: 9 of the 12 patients were included in the group bearing the aforementioned lesion, the remaining 3 in a second group, not characterized by an involvement of a frontal lobe cerebral lesion. The functional outcome of the 12 patients was then analyzed in light of the presence, or not, of a frontal cerebrovascular lesion, to investigate the neuromotor and neuropsychological effects.

\section{Results}

\section{Influence of the type of cerebrovascular accident on motor outcome}

From an evaluation of the trend of the mean values of the neuromotor scales, a parametrically but not statistically significant modification of the results obtained was observed by measuring the MI ULl, MI LLl and TCT scales in the group with hemorrhagic brain injury. The analysis of variance did not show any statistical significance in the relationship between the type of stroke (ischemic, hemorrhagic or both) and the motor impairment highlighted by our 12 patients passing from time T0 to time T1 (Table 2 and Figures 2,3).

\section{Influence of cerebral lesion type on the neuropsychological scales results}

The analysis of variance did not reveal a statistically or 


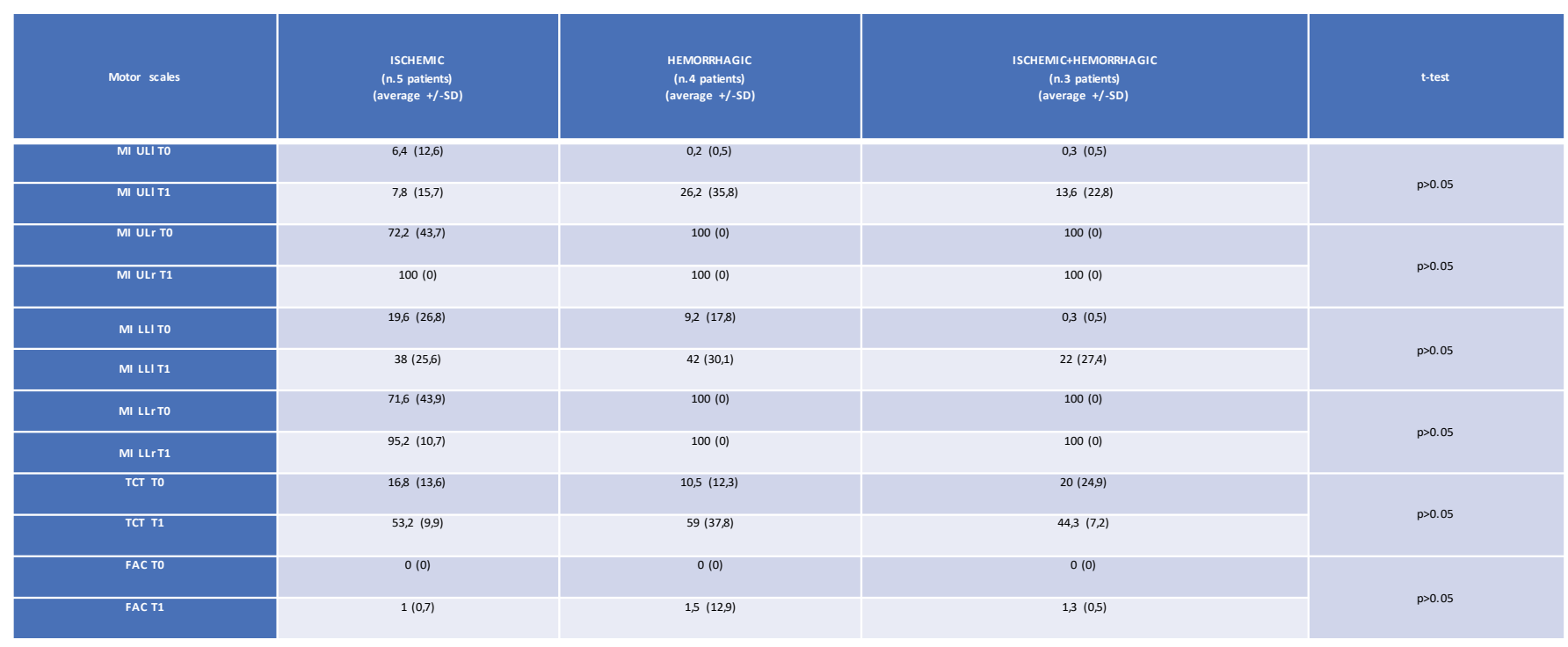

Table 2: The average and standard deviation of the motor scales (at T0 and T1) of the 12 patients, referred to the type of cerebral lesion. MI: Motricity Index; UL: upper limb; LL: lower limb; I: left; r: right; TCT: Trunk Control Test; FAC: Functional Ambulation Category (own elaboration).

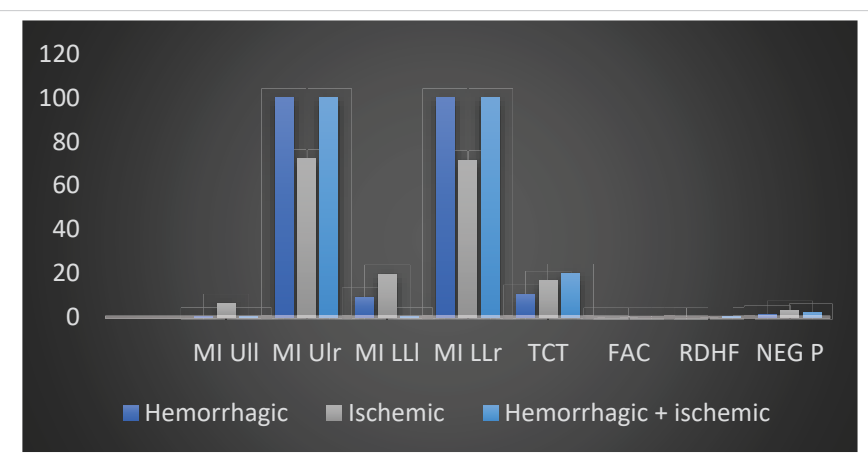

Figure 2: The trend of mean values of motor and neuropsychological scales as a function of the type of brain injury found in the 12 patients of the sample at time T0 (own elaboration).

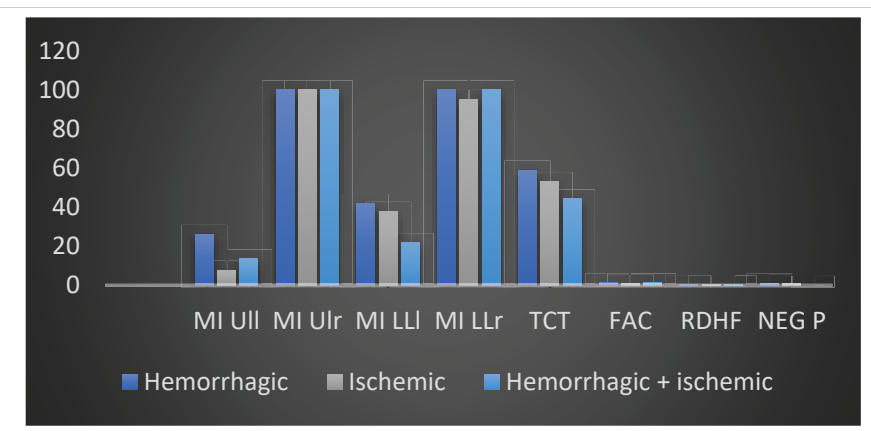

Figure 3: The trend of mean values of motor and neuropsychological scales as a function of the type of brain injury found in the 12 patients of the sample at time T1 (own elaboration).

parametrically significant relationship between the type (ischemic, hemorrhagic and ischemic + hemorrhagic) of cerebral stroke and the variations of the neuropsychological profile of our 12 patients; this absence of significance was found both at time T0 and time T1 (Table 3 and Figures 2,3).

Influence of the cerebral lesion site on the motor scales results

In the group of 12 patients, the T-Student test showed statistically significant changes in the importance of the lesion site in defining the degree of motor disability. In particular, we observed, about the presence of frontal lobe lesions, a statistically significant variation passing from the T0 time to the T1 time for the following motor scales in 9 of 12 recruited patients: MI LLl (26.4 vs. 62, with $p<0.05)$, TCT (43.6 vs. 80.6, with $p<0.01$ for equal variance assumed and $p<0.05$ for equal variance not assumed), FAC (0.8vs. 2.3 with $p<0,01$ for equal variance assumed and $p<0.05$ for equal variance not assumed) (Table 4 and Figures 4,5).

\section{Influence of the cerebral lesion site on the neuropsycho- logical scales results}

The T-Student test showed no statistically significant variation, depending on the involvement of the frontal lobe, passing from time $\mathrm{T} 0$ to time $\mathrm{T} 1$ of the neuropsychological profile evaluated with RDHF scales ( 0.3 vs. 0.6 , with $p>0,05$ ) and NEG P (0.7 vs. 1, with $p>0.05$ ) (Table 5 and Figures 4,5).

\section{Discussion}

A critical analysis of the literature shows that cerebrovascular accidents represent one of the major causes of morbidity in many countries [18]. Within the neurocognitive disorders secondary to a cerebral apoplectic event, unilateral spatial neglect, or neglect, is the one with the greatest disabling impact on the quality of life and the prospect of functional recovery of the patient [19].

Using an analysis of the parametric variance as a function of the time factor (time T0 and T1), we investigated the degree of relationship between the type of cerebrovascular accident of each patient recruited (ischemic, hemorrhagic, ischemichemorrhagic) and the degree of neuromotor disability of the same. In contrast to the current literature [20-22], no statistically significant variations were observed in the 


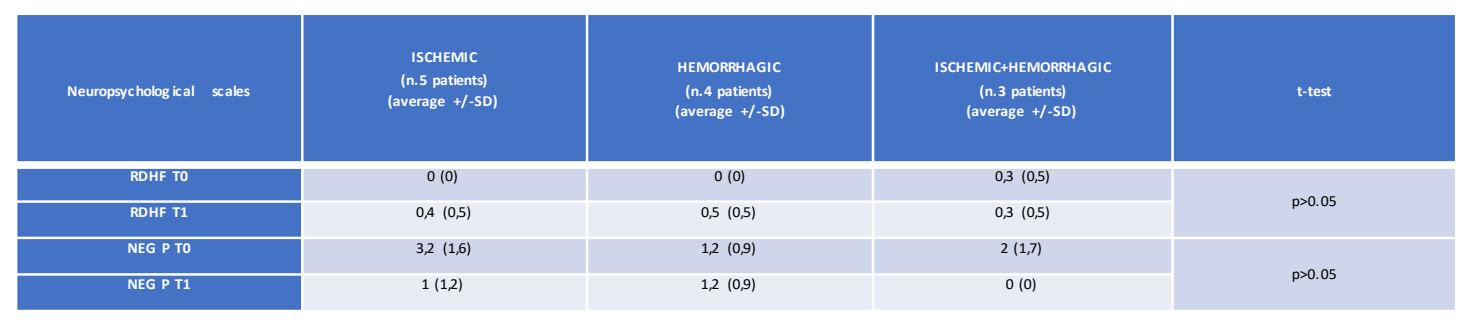

Table 3: The average and standard deviation of the neuropsychological scales (at T0 and T1) of the 12 patients, referred the cerebral lesion typology (own elaboration). RDHF: representational drawing of the human figure. NEG P: personal neglect.

\begin{tabular}{|c|c|c|c|}
\hline Motor scales & $\begin{array}{l}\text { Neuroradiolog ical framework with FRONTAL lobe } \\
\text { interest ( } n^{\circ} 9 \text { patients) }\end{array}$ & $\begin{array}{l}\text { Neuroradiolog ical framework without FRONTAL } \\
\text { lobe interest }\left(n^{\circ} 3 \text { patients) }\right.\end{array}$ & t-test \\
\hline MI ULI To & $3,5(9,5)$ & $0,6(0,5)$ & \multirow{2}{*}{$p>0.05$} \\
\hline MI ULI T1 & $8,7(16,6)$ & $35,3(37,8)$ & \\
\hline MI ULr TO & $84,5(34,2)$ & $100(0)$ & \multirow{2}{*}{$p>0.05$} \\
\hline MI ULr T1 & $100(0)$ & $100(0)$ & \\
\hline мI LL то & $6,7(19,2)$ & $25(20,8)$ & \multirow{2}{*}{$P<0.05^{*}$} \\
\hline MI LLI T1 & $26,4(23,6)$ & $62(12,4)$ & \\
\hline MI LLrTo & $84,2(34,5)$ & $100(0)$ & \multirow{2}{*}{$p>0.05$} \\
\hline MI LLrT1 & $97,3(8)$ & $100(0)$ & \\
\hline тCT то & $14,6(17,7)$ & $18(6)$ & \multirow{2}{*}{$\begin{array}{l}p<0.01^{*} \text { for equal variance assumed } \\
p<0.05^{*} \text { for equalvariance not assumed }\end{array}$} \\
\hline TCT T1 & $43,6(13,5)$ & $80,6(17,4)$ & \\
\hline FAC TO & $0(0)$ & $0(0)$ & \multirow{2}{*}{$\begin{array}{l}p<0.01^{*} \text { for equalvariance assumed } \\
p<0.05^{*} \text { for equalvariance not assumed }\end{array}$} \\
\hline FAC T1 & $0,8(0,6)$ & $2,3(0,5)$ & \\
\hline
\end{tabular}

Table 4: Average and standard deviation of the motor scales (at T0 and T1) of the 12 patients regarding the presence/absence of involvement of the frontal lobe (own elaboration).

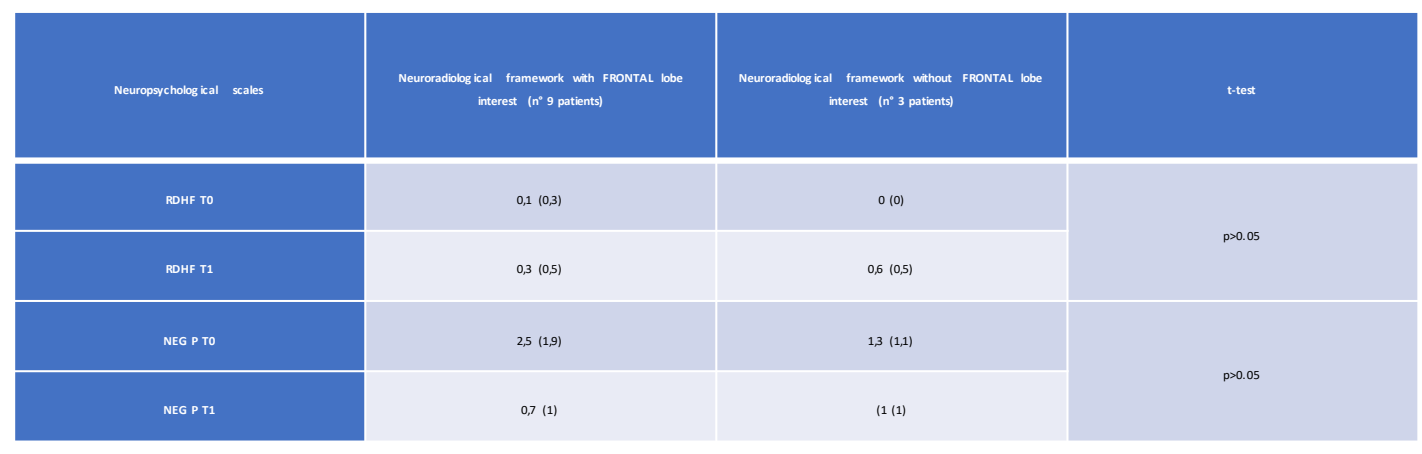

Table 5: Mean and standard deviation of the neuropsychological scales (at T0 and T1) of the 12 patients with reference to the presence / absence of involvement of the frontal lobe (own elaboration).

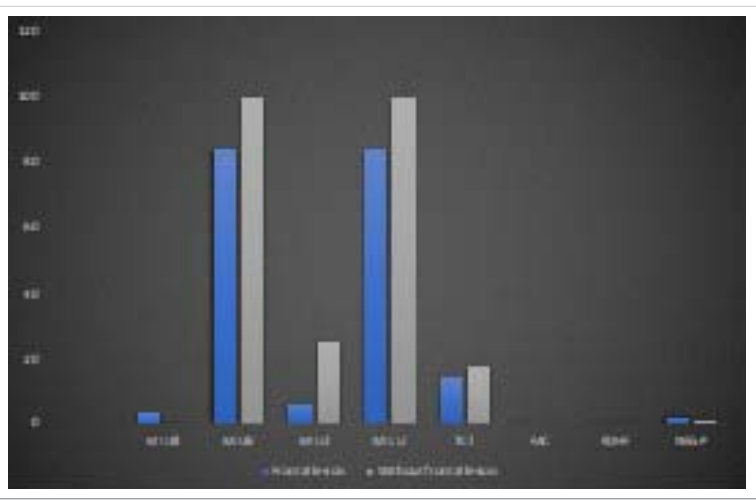

Figure 4: Trend of the mean values of the motor and neuropsychological scales as a function of the presence and not of a frontal lesion at time TO in the 12 recruited patients (own elaboration).

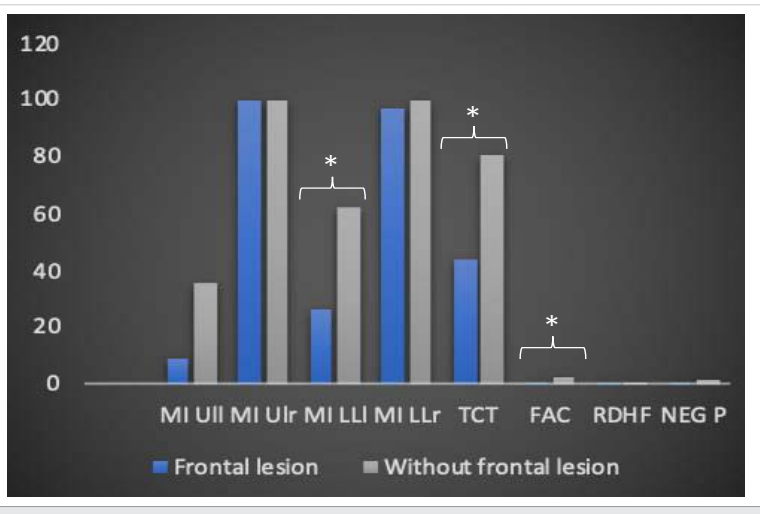

Figure 5: The trend of the average values of the motor and neuropsychological scale as a function of the presence and not of a frontal injury at time $\mathrm{T} 1$ in the 12 patients recruited (own elaboration). *Significant variation trend 
clinical-functional parameters used for the quantification of neuromotor impairment (going from time T0 to T1) in all patients undergoing the proposed rehabilitation treatment. Several hypotheses justify this conclusion: a. the variance between the data compared to the time factor is "zero" or "very low" and therefore statistically insignificant; b. a number of our study sample, significantly lower than that of authoritative works existing in the literature [20-22]; c. type of assessment tools of the neuromotor deficit used.

In the same way, using the analysis of the variance of neuropsychological scales, the relationship between the type of stroke (ischemic, hemorrhagic, ischemic-hemorrhagic) and the alteration of body representation in the survey sample was investigated. The analysis of variance did not reveal statistically significant changes in the assessment parameters of the "neuropsychological impairment", an observation that can be generalized both to the results analyzed at time T0 and those examined at time $\mathrm{T} 1$. The present result seems to be in antithesis with what reported in the study of Chwen-Yng, et al. [23] in which it is underlined how in hemorrhagic brain lesions patients show a more lateralized profile of impairment compared to patients with ischemic damage. Also, in this case, the difference found between the results of our study and those of the literature could be attributable, in the first instance, to diversity in the sample size as well as to the use of different neuropsychological evaluation scales. Using the T-Student parametric test, the influence of the involvement of the right frontal lobe on the degree of neuromotor and neuropsychological disability of the 12 patients recruited was analyzed. The T-Student test did not reveal statistically significant differences either at the level of the personal neglect scale or within the "Representational Drawing" concerning the involvement of the right frontal lobe; it is clear that the absence of a relationship, between the presence of a frontal lesion and the modification of the neuropsychological scales, remains unchanged over time; in fact, the statistical insignificance of the above results is found both at time T0 and at time T1. The same T-Student test instead showed statistically significant changes in the assessment parameters of neuromotor disability, concerning the presence of a neuroradiological lesion profile including the right frontal lobe and the time factor: the scales for which statistically significant variations were detected at time $\mathrm{T} 1$ are the MI for the left lower limb, the TCT, and the FAC. This evidence is in agreement with the studies contained in the literature concerning cases of hypokinetic directional neglect secondary to a lesion of the right frontal lobe $[24,25]$ and deficit in planning and beginning of the movement [24]. Based on what has been shown by Heilman, et al. [26] directional hypokinesia, due to which a motor deficit in the use of the ipsilesional limb in the contralesional space is observed, implies a disability at the level of the motor programming process. The processing, as well as the motivation, the execution, the coding of the intensity of the force and the direction of the movement, are implemented by the primary motor cortex, anatomically localized in correspondence of the frontal lobe. The worst results obtained by patients with frontal injury at time T1 in MI for the left lower limb, in TCT, and in FAC, compared to those who did not have involvement of the aforementioned lobe, can be justified on the basis of what has just been said. Since the primary motor cortex is anatomically localized in the frontal lobe, a lesion affecting the latter can affect movement in its components, be they motivational, elaborative, executive, directional or force coding; in addition to the tasks to which the primary motor cortex is used, it is important to emphasize that the non-significance of the results inherent to MI for the left upper limb can be traced to the somatotopic distribution of the representations of the body districts at the level of this brain region; in relation to the topography of the representations, it is possible to hypothesize that according to the localization of the lesion at the frontal lobe, different portions of the cerebral cortex are affected and, consequently, different body areas. The results reveal a statistically significant involvement of the lower limbs (MI left limb and FAC) and of the trunk (TCT), with savings in the left upper limb, compatible with frontal brain injuries and the primary motor area.

\section{Conclusion}

In light of the results observed in the following study, we have confirmed the importance of the anatomicaldysfunctional correlation as a key concept from which to start in any neurorehabilitative treatment approach.

Our work has highlighted the basic role of the right frontal lobe in the programming and execution of the gesture and its kinesthetic control as regards the left lower limb and the trunk.

What emerged from the following study represents a data of fundamental importance in the field of neurorehabilitation, since the motivation, the execution, the coding of the intensity of the force and the direction of the movement, parameters constituting the kinesthetic control of the gesture, are mediated by the primary motor cortex anatomically located in the frontal lobe

Investigating the degree of relationship between the type of cerebrovascular accident of each patient recruited (ischemic, hemorrhagic, ischemic-hemorrhagic) and the degree of neuromotor disability of the same, no statistically significant variations were observed in the clinical-functional parameters used for the quantification of neuromotor impairment in all patients undergoing the proposed rehabilitation treatment; similarly, the relationship between the type of stroke (ischemic, hemorrhagic, ischemic-hemorrhagic) and the alteration of body representation in the survey sample did not reveal statistically significant changes in the assessment parameters.

From a careful self-critical analysis of the data collected 
in our study, some irrefutable limits emerge. First of all, it is evident that the variance between the data with respect to the time factor was equal to "zero" or in any case "very low" and therefore statistically insignificant for the purpose of a concrete validation of the results achieved; in the second analysis, the small number of our study sample did not allow us to give a concrete statistical value to our work, underlining the possible weakness of the parametric analysis used by us; lastly, we cannot exclude that the outcome parameters used are not among the most suitable in supporting the purpose of our study.

Further studies capable of recruiting larger study samples, with the structuring of a study model that can make use of additional outcome measures and greater statistical strength, represent the challenge for all those who make anatomicaldysfunctional correlations the basis on which to plan increasingly innovative integrated rehabilitation treatment strategies capable of improving the quality of life of the patient suffering from stroke outcomes.

\section{References}

1. Blanke 0.1 and me: self-potraiture in brain damage. Front Neurol Neurosci. 2007; 22: 14-29.

Pubmed: https://www.ncbi.nlm.nih.gov/pubmed/17495503

2. Gallagher S, Cole J. Body schema and body image in a deafferented subject. J Mind Behaviour. 1995; 16: 369-390.

3. Shildler P. The image and appearance of the human body. 1935 . Routledge. London.

4. Maravita A, Iriki A. Tools for the body (schema). Trends Cogn Sci. 2004; 8:79-86.

Pubmed: https://www.ncbi.nlm.nih.gov/pubmed/15588812

5. Berlucchi G, Aglioti S. The body in the brain: neural bases of corporeal awareness. Trends Neurosci. 1997; 20: 560-564.

Pubmed: https://www.ncbi.nlm.nih.gov/pubmed/9416668

6. Maravita A, Spence C, Driver J. Multisensory integration and the body schema: close to hand and within reach. Current Biology. 2003; 13: R531-539.

Pubmed: https://www.ncbi.nlm.nih.gov/pubmed/12842033

7. Coslett HB, Saffran EM, Schwoebel J. Knowledge of the human body: a distinct semantic domain. Neurology. 2002; 59: 357-363.

Pubmed: https://www.ncbi.nlm.nih.gov/pubmed/12177368

8. Jeannerod M, Biguer B. Egocentric reference and represented space. Revue Neurologique (Paris). 1989; 145: 635-639.

Pubmed: https://www.ncbi.nlm.nih.gov/pubmed/2682938

9. He BJ, Shulman GL, Snyder AZ, Corbetta M. The role of impaired neuronal communication in neurological disorders. Curr Opin Neurol. 2007; 20: 655-660.

Pubmed: https://www.ncbi.nlm.nih.gov/pubmed/17992085

10. Ptak R, Schnider A. The attention network of the human brain: relating structural damage associated with spatial neglect to functional imaging correlates of spatial attention. Neuropsychologia. 2011; 49: 3063-3070.

Pubmed: https://www.ncbi.nlm.nih.gov/pubmed/21787795

11. Starkstein SE, Fedoroff JP, Price TR, Leiguarda R, Robinson RG. Anosognosia in patients with cerebrovascular lesions. A study of causative factors. Stroke. 1992; 23:1446-1453.

Pubmed: https://www.ncbi.nlm.nih.gov/pubmed/1412582

12. Maeshima S, Truman G, Smith DS, Dohi N, Nakai K, et al. Is unilateral spatial neglect a single phenomenon? J Neurol. 1997; 224:412-417. Pubmed: https://www.ncbi.nlm.nih.gov/pubmed/9266458

13. Evyapan D, Kumral E. Pontine anosognosia for hemiplegia. Neurology. 1999; 3: 647-649.

Pubmed: https://www.ncbi.nlm.nih.gov/pubmed/10449140

14. Pia L, Neppi-Modona N, Ricci R, Berti A. The anatomy of anosognosia for hemiplegia: a meta-analysis. Cortex. 2004; 40: 367-377. Pubmed: https://www.ncbi.nlm.nih.gov/pubmed/15156794

15. Berti A, Bottini G, Gandola M, Pia L, Smania N, et al. Shared cortical anatomy for motor awareness and motor control. Science. 2005; 309: 488-491.

Pubmed: https://www.ncbi.nlm.nih.gov/pubmed/16020740

16. Karnath HO, Baier B, Naegele T. Awareness of the functioning of one's own limbs mediated by insular cortex? J Neurosci. 2005; 25: 71347138.

Pubmed: https://www.ncbi.nlm.nih.gov/pubmed/16079395

17. Jourdan S, Torres C, Agostino F, Chiapolini S, Morin L, et al. Effets de la reeducation sur l'héminégligence. Kinésithérapie. 2010; 107: 28-33.

18. Luauté J, Schwartz S, Rossetti $Y$, Spiridon $M$, Rode G, et al. Dynamic changes in brain activity during prism adaptation. J Neurosci. 2009; 29: 169-178.

Pubmed: https://www.ncbi.nlm.nih.gov/pubmed/19129395

19. Buxbaum LJ, Ferraro MK, Veraminti T, Farné A, Whyte J, et al Hemispatial neglect-subtypes, neuroanatomy and disability. Neurology. 2004; 62:749-756.

Pubmed: https://www.ncbi.nlm.nih.gov/pubmed/15007125

20. Paolucci S, Antonucci G, Grasso MG, Bragoni M, Coiro P, et al. Functional outcome of ischemic and hemorrhagic stroke patients after inpatient rehabilitation - a matched comparison. Stroke. 2003; 34: 2861-2865. Pubmed: https://www.ncbi.nlm.nih.gov/pubmed/14615613

21. Lauretani F, Saccavini M, Zaccaria B, Agosti M, Zampolini M, et al. Rehabilitation in patients affected by different types of stroke. A oneyear follow-up study. Eur J Phys Rehabil Med. 2010; 46: 511-517. Pubmed: https://www.ncbi.nlm.nih.gov/pubmed/20414185

22. Lipson DM, Sangha H, Foley NC, Bhogal S, Pohani G, et al. Recovery from stroke: differences between subtypes. Int J Rehabil Res. 2005; 28: 303-308.

Pubmed: https://www.ncbi.nlm.nih.gov/pubmed/16319555

23. Chwen-Yng S, Jyh-Jong C, Hui-Mei C, Chin-Jung S, Tsui-Hsien C, et al. Perceptual differences between stroke patients with cerebral infarction and intracerebral hemorrhage. Arch Phys Med Rehabil. 2000; 81: 706714.

Pubmed: https://www.ncbi.nlm.nih.gov/pubmed/10857510

24. Bottini G, Sterzi R, Vallar G. Directional hypokinesia in spatial hemineglect: a case study. J Neurol Neurosurg Psychiatry. 1992; 55 : 562-565.

Pubmed: https://www.ncbi.nlm.nih.gov/pmc/articles/PMC489166/

25. Sapir A, Kaplan JB, He BJ, Corbetta M. Anatomical correlates of directional hypokinesia in patients with hemispatial neglect. $J$ Neurosci. 2007; 15: 4045-4051.

Pubmed: https://www.ncbi.nlm.nih.gov/pubmed/17428982

26. Heilman KM, Bowers D, Coslett HB, Whelan H, Watson RT. Directional hypokinesia: prolonged reaction times for leftward movements in patients with right hemisphere lesions and neglect. Neurology. 1985; 35 : 855-859. Pubmed: https://www.ncbi.nlm.nih.gov/pubmed/4000486 